\title{
The process development challenge for a new vaccine
}

\author{
Barry C Buckland
}

The challenges of vaccine development are not limited to identification of suitable antigens, adjuvants and delivery methods, but include regulatory, technical and manufacturing hurdles in translating a vaccine candidate to the clinic. Process development is the technological foundation that underlies the manufacture of new vaccines and is central to successful commercialization.

Public demand for safe and effective vaccines continues to be strong. Despite this need and in contrast to pharmaceuticals (small-molecule drugs) or to therapeutic proteins, only four (Merck \& Co., Inc., GlaxoSmithKline, Wyeth and Sanofi Pasteur) of the major pharmaceutical companies have the knowledge and facilities required to develop and manufacture a new vaccine product candidate. Recently, in response to demand for innovative new vaccines, smaller companies are emerging with vaccine development capabilities. These companies include Chiron, Berna Biotech, Acambis and Baxter. The challenges facing a new entrance in this arena are formidable: the complexity of the technology, the need for expensive specialized facilities and the demanding regulatory requirements. The greatest hurdle is translating basic science advances into real vaccines that can be produced in adherence to stringent regulatory requirements on a sufficient scale to have a meaningful public health impact. For a vaccine with wide utility, the manufacture of tens of millions of doses per year is required. The broad quality goal is that each and every dose is equivalent, safe and effective. The regulatory burden is the level of proof and documentation necessary to provide guarantees that this broad quality goal is achieved on an ongoing basis.

The central issue related to technology is the evolution and translation from a procedure

Merck Research Laboratories, West Point,

Pennsylvania 19486, USA.

e-mail: barry_buckland@merck.com

Published online 5 April 2005; doi:10.1038/nm1218 used for making vaccines in a basic research laboratory to a process that can be scaled up and run reproducibly in a manufacturing environment to make tens of millions of doses per year. There are many academic and governmental research laboratories and fledgling biotechnology companies with the ability to innovate and generate interesting new vaccine product candidates, which is, of course, the first key step in the vaccine development process. But this stage is just the beginning of a cycle of intense activity that requires great skill, sophisticated facilities, determination and much patience.

\section{Regulatory considerations}

The complicated process of technology development needs to be implemented within an environment of regulatory oversight that is, in a number of ways, much more stringent than those governing the manufacture of either therapeutic proteins or small-molecule pharmaceuticals. The basis for this increased stringency derives in part from the fact that vaccines are much more difficult to characterize analytically than many pharmaceuticals. Simply put, a small-molecule drug has a molecular weight of less than 1,000, analytical analysis can provide complete assurance of its identity and composition and the final product is dosed based on its weight. In contrast, for example, the virus-like particle (VLP) used as the basis for a vaccine against the human papillomavirus is more than 10,000-fold greater in size than a small-molecule drug and the dose is specified based on both weight and biological potency. Because of the challenge of analysis of such a large molecule, there has been a historic tendency in development that the process of producing the vaccine itself defines the product, which has lead to a level of regulatory oversight that is much greater than for a small-molecule pharmaceutical. Furthermore, any changes in either scale or process require reassurance that the product will remain unchanged in terms of its efficacy and safety and satisfactory proof is demanded by leading regulatory authorities. Such bridging for a change in process or scale would be accompanied by very sophisticated and extensive analytical characterization, and perhaps the requirement for additional clinical testing simply to prove equivalence between the new and the old vaccine.

Importantly, the demand for safety of any vaccine is also unusually high, driven by the fact that vaccines are injected into the bodies of healthy children and adults so as to prevent diseases, rather than the typical drug that is used to treat individuals suffering from diseases. As such, the risk-benefit ratio for vaccines rightly emphasizes the importance of very well-characterized and very safe vaccines. The resulting strict regulatory oversight impacts every step in the cycle from the testing of the cell line used to produce the vaccine to the testing of the final vaccine product. All vaccine manufacturing processes must be described and characterized in great detail, including the nature and performance of the specific equipment used for every step of the manufacturing process. These requirements create an essential rigidity in approach which is unforgiving of an error in judgment. As a result, any misstep along the way can result in a very time-consuming (sometimes taking years) and expensive correction in the manufacturing process. 


\section{Vaccine development}

Human papillomavirus. The complex challenges inherent in the translation of a basic vaccine concept into a real vaccine product can be illustrated by some recent examples from the Merck Research Laboratories for three vaccine product candidates. These projects have been underway for at least 7 years and are well advanced in their development. In fact, it is anticipated that they will all be filed for licensure in the United States and in many other countries in 2005. The first of these is a vaccine against four types of human papillomavirus (HPV): 6, 11,16 and 18. This product candidate was licensed from Commonwealth Serum Laboratories (Australia) and the University of Queensland after very encouraging extensive animal studies using VLPs_produced by a process of self-assembly of the HPV L1 protein. These early studies provided a promising preclinical 'test of concept' ${ }^{1}$ supporting the potential of this approach for development as a prophylactic vaccine for humans. The choice of VLP as antigen was attractive because the product is inherently noninfectious and also because of the excellent track record with vaccines for hepatitis B based on VLPs ${ }^{2}$. The preclinical-to-clinical translation process for the HPV vaccine began in 1993, and came to fruition 9 years later ${ }^{3-5}$.

The second critical step in the process was to make a vaccine for rapid evaluation in the clinic (assessed by its ability to generate antibodies against the HPV virus), recognizing that the method used to produce the vaccine would be crucial and would set the stage for all subsequent phases of the developmental program. To prepare the vaccine for initial clinical trials, a strategy was adopted that would be similar to one conceptually planned for eventual full-scale manufacturing once the vaccine was licensed. A crucial starting point was the development of a cell-based expression system that was stable over many generations and produced large amounts of high-quality VLPs. The decision to use Saccharomyces cerevisiae as the expression host for intracellular VLPs was excellent in this regard because the yeast can readily be grown in fermentors of several thousand liters and reproducibly forms intracellular VLPs during its growth cycle. The major reason that this particular host cell was chosen was because of the extensive experience at the Merck Research Laboratories using $S$. cerevisiae to make a successful hepatitis B vaccine (Recombivax). It was possible to finalize choice of this yeast strain based on productivity, reproducibility and the specific fermentation conditions at an early stage of the program, thus allowing process development to focus on scale-up, purification, formula-

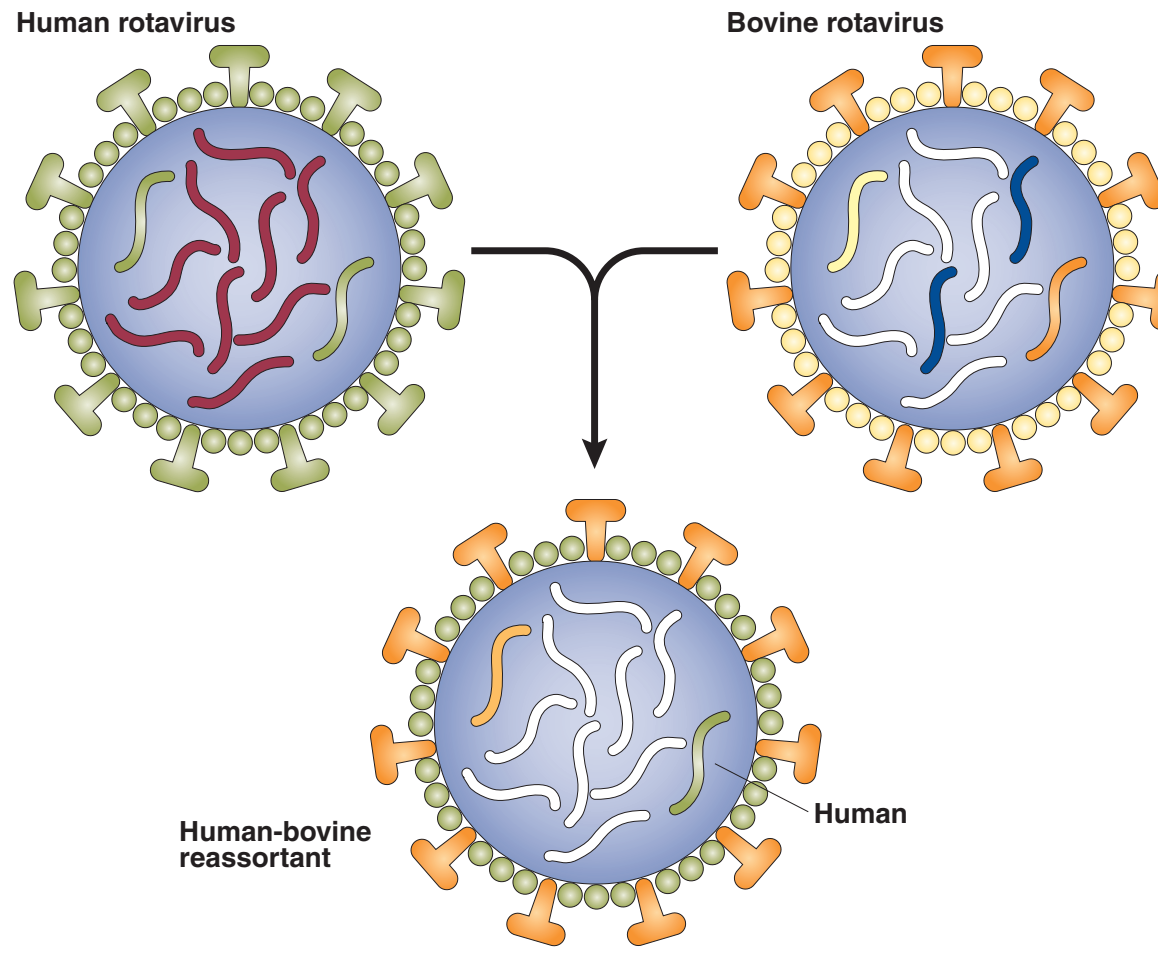

Figure 1 Rotavirus reassortant to generate oral live virus vaccine. RotaTeq is a polyvalent vaccine consisting of five human-bovine reassortants: four G serotypes (G1, G2, G3, G4) representing $80 \%$ of the $G$ strains circulating worldwide, and one $P$ serotype representing $>75 \%$ of the $P$ strains circulating worldwide.

tion and the analytical methods required for product characterization and potency measurements.

Once the selection of the expression system to be used in vaccine manufacture was made, a master seed bank was established from the engineered yeast strain; this bank was sufficient in quantity for use throughout the duration of the program. A unique VLP was made for each of the four HPV types included in the vaccine, requiring technology development for the fermentation process, cell harvesting, cell disruption, cell debris removal, chromatography-based purification and absorption to the adjuvant of choice for each VLP to make the final formulated product. The VLPs are stable for several years under refrigerated conditions and the aluminum-based adjuvant was chosen to be the same as the Recombivax hepatitis B vaccine. Analytical methods based on antibody binding to the functional epitopes of the VLPs were developed to quantify the potency of the vaccine (in vitro using an enzyme-linked immunoassay) and that were sufficiently precise to be able to monitor VLP stability when stored as a refrigerated final product for several years, and also separately quantify each VLP representing the specific HPV types contained in the four-component vaccine. The challenge is to choose an anti- body that recognizes the functional epitope for the HPV strain in question and which is not subject to interference from the other three HPV types present in the vaccine. Other key tests were also developed to search for impurities, such as highly sensitive assays to detect trace amounts of the undesired yeast cell proteins.

As mentioned earlier, a major challenge in commercial vaccine manufacturing is to ensure that the process is scalable so that it is possible to build a factory for tens of millions of doses, and that the vaccine made at this scale behaves the same way in the clinic as vaccine made at a much smaller scale earlier in the program. In addition, the analytics need to be sufficiently well developed so that the precise definition of potency at a particular point in the development cycle will not change as the program progresses. Specifically, it is essential to be able to link the clinical performance, comparing the vaccine made at an early stage of development to that used for final stage development and testing in the clinic. In the case of the HPV vaccine program, the vaccine used in the phase 1 clinical trials was manufactured in a pilot plant (a pilot plant uses the same type of equipment as the final factory) using a less-developed version of the production process and at a smaller scale of operation 


\section{COMMENTARY}

(300 liters). The bulk preparation of phase 3 vaccine was made at full-scale manufacturing, which is within a factor of 10 of pilot-scale manufacturing.

So, what essential components are needed for the successful development of a large-scale manufacturing process such as for the HPV vaccine described here? First, facilities are required to produce representative clinical supplies at the pilot scale. Second, a manufacturing facility needs to be designed and constructed. These facilities historically have been built by the company that develops the vaccine. Third, vaccine supplies need to be made within this manufacturing facility before licensure, and usually representative doses need to be tested in the clinic as part of the required program to gain approval of the vaccine for licensure. Fourth, sophisticated analytical characterization is required so that a rationale can be developed for making small changes in the process or changes in scale of production and bridging without the need for confirmation in the clinic. Fifth, a clinical research team needs to be able to coordinate large and complicated clinical trials as well as the development of serological assays that are used as a surrogate for protection. Sixth, the regulatory expectations for filing throughout the world need to be fully understood early in the process development effort. To carry out these diverse and complex development tasks, a committed team of approximately ten or more people is required to lead and coordinate such an inherently complicated project; this team would include representatives from the process development group, the analytical group, the clinical team, manufacturing, marketing, and from the internal regulatory organization. Patience and determination are needed, often for 10 years or more, to complete this cycle. After all this effort, the end product is $0.5 \mathrm{ml}$ of safe and efficacious vaccine in a vial or syringe containing purified VLPs of four different HPV types at a precise and reproducible dose for each along with adjuvant.

Rotavirus. The vaccine being developed for rotavirus is a live attenuated virus vaccine that contains five different human-bovine virus reassortants ${ }^{6,7}$, which have been adapted to large-scale production using VERO cells (Fig. 1). Rotavirus is a member of the Reovirus genus in the Reoviridia family of viruses. The final reassorted strain was selected from each of the five final types by taking advantage of the natural capability of the rotavirus genes to reassort in cell culture.

The general outline described for HPV would apply in terms of the approach for developing a vaccine but the technologyrelated issues are very different. The process used for rotavirus vaccine requires masterseed development for each of the five rotavirus reassortants as well as for the VERO cell bank. There are five different processes involved, with a separate process for each reassortant. The VERO cells are grown to confluence on a surface and then infected with rotavirus. The product is a live virus (initially developed at the Children's Hospital of Philadelphia) that needs to be formulated in a manner that maintains good potency in the refrigerator for each of the five assortants. The route of administration is oral. Potency measurements are based on quantification of attenuated live virus using PCR technology, which is used to quantify each of the individual five reassortants in the final formulated vaccine. The clinical trials required for this vaccine to prove both safety and efficacy have been very large and expensive, requiring more than 100,000 of vaccine doses made by a process representative of final-scale manufacturing (Fig. 2).

Varicella zoster. In the last example, the vaccine under development for postherpetic neuralgia or shingles (caused by reactivation of latent varicella zoster virus infections) is based upon Merck Research Laboratories' existing live attenuated chicken pox vaccine. The shingles vaccine is being developed for older adults, whereas the chicken pox vaccine is for young children. For increased immunogenicity in older individuals, the dose of the vaccine administered is much greater than that used to immunize children against chicken pox. Given that the requisite dose of vaccine is higher for use in adults for prevention of shingles, and that varicella virus is inherently difficult to grow to high titers in culture, significant obstacles

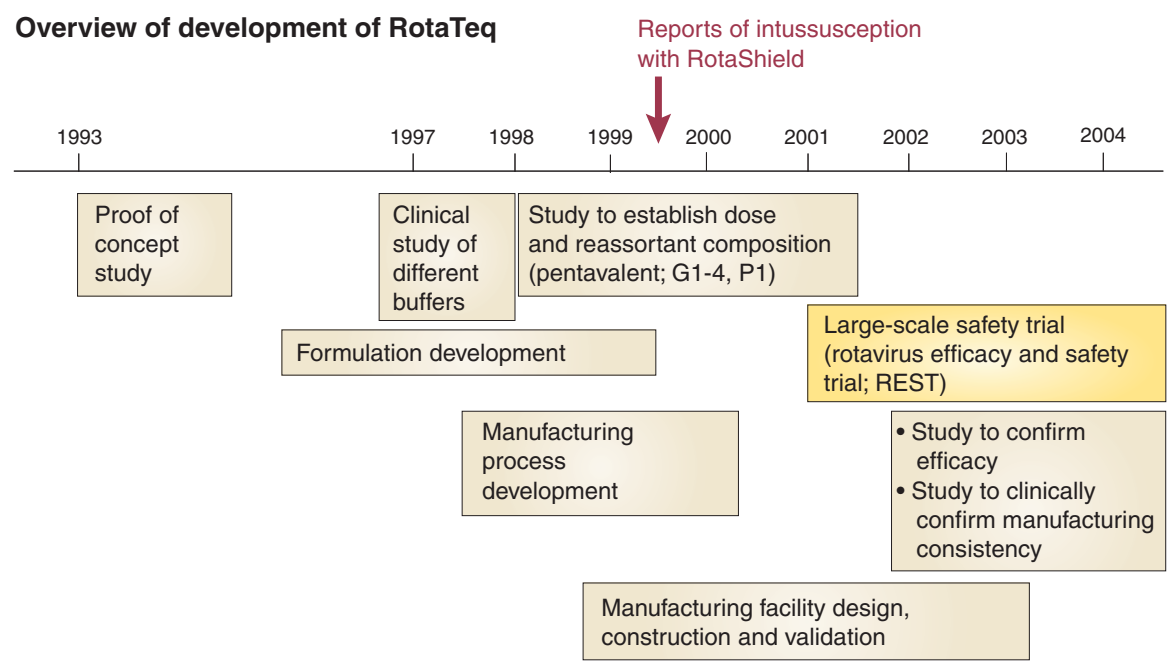

Figure 2 Overview of the development of a vaccine against rotavirus (RotaTeq). G1-4, P1, rotavirus serotypes. RotaShield, a vaccine for rotavirus developed by Wyeth. had to be overcome to manufacture sufficient quantities of vaccine virus. Further, as the virus is unstable at normal temperatures, a unique technical challenge was to develop a method to preserve its stability using lyophilization in the presence of specially developed preservatives to permit an acceptable shelf life.

Again, the manufacturer must ensure that each dose of the vaccine is safe and effective. In the case of the varicella postherpetic neuralgia vaccine, formulation required knowledge of process capabilities, precision of measurement and stability assumptions. An adequate dose range must be evaluated in the clinic. For safety, the key is to obtain data representative of a vial soon after filling in order to reflect the key is to evaluate a dose representative of a vial held until the end of the claimed shelf life (i.e., when the dose will be lower as a result of loss of infectious virus upon storage). This example illustrates integration between process development, manufacturing and clinical evaluation.

The development cycle of vaccine generation is a complex and expensive undertaking, requiring the rapid development of a scalable process using very divergent technologies. Depending on the type of product, vaccines may be made from live viruses, microbially expressed proteins (including microbially expressed VLPs), polysaccharides, polysaccharides conjugated to a carrier protein, inactivated virus or DNA. Presently there are only a few groups in the world positioned to rapidly develop a vaccine because of the unique skills required - an effective team would be composed of both scientists and bioprocess engineers who together could quickly develop

construction and validation the highest dose. For efficacy determination, 
a practical, robust and scalable process built upon a solid understanding of the biology. Finally, there is the issue of the complexity of the analytics both for potency determination as well as serology analysis. A company with this capability has an enormous competitive advantage because of the manner in which technology issues can dominate vaccine development. Inadequate process development can lead to a vaccine that is not scalable and that may also be variable, leading to extended development cycle times and unacceptable costs.

The central issue of technology complexity is addressed in The Development Factory by Gary Pisano ${ }^{8}$. Specifically, he addresses the strategic leverage of process development capabilities as applied to pharmaceuticals and biotechnology, and these basic points are even more applicable to vaccines. Pisano's research concluded that process innovation can be the key to competitive edge-behind the success of many new products, especially in the biotechnology industry, lies the development of new process technologies that provide a broad array of crucial benefits. These include lower capital costs, reduced time to market, improved quality, increased flexibility and lower operating costs. In the new environment of increased regulation and demand for lower costs, process development has quietly become an important part of competing, especially in the realm of vaccine development, where processes are exceedingly complicated and regulatory oversight is extensive.

The challenge for the vaccine community in the near future is to better balance the equation between risk and benefit depending on the intended application. For example, a vaccine intended for universal pediatric use would require proof of efficacy as well as very extensive testing for safety. This would be in contrast to a vaccine being developed against a predicted bioterrorism threat, in which case use may only occur in an emergency situation and testing for efficacy may not be possible in humans. In the latter example, a simpler and more rapid path toward approval might be considered more appropriate.

In the manufacture of new vaccines, process development must occur in parallel with the development of advanced analytical methods for vaccine characterization and determination of vaccine potency. These activities need to be fully integrated with a plan for evaluation in the clinic, for obtaining regulatory approval and for manufacturing. The process develop- ment scientist or engineer must be cognizant of all these related issues to successfully provide the technology bridge between the interesting new research lead and manufacture of the final product - thereby laying the groundwork toward eventual licensure and launch of a new vaccine.

\section{ACKNOWLEDGMENTS}

The author would like to thank M. Feinberg of Merck Research Laboratories for his comments.

\section{COMPETING INTERERESTS STATEMENT}

The author declares competing financial interests (see the Nature Medicine website for details).

Published online at http://www.nature.com/ naturemedicine/

1. Jansen, K.U. et al. Vaccine 13, 1509-1514 (1995).

2. Knicksen, P.J. \& Miller, W.J. in Vaccines: New Approaches to Immunological Problems (ed. Ellis, R.W.) 177-204 (Butterworth-Heinemann, Stoneham, Massachusetts, 1992).

3. Brown D.R. et al. Vaccine 22, 2936-2942 (2002).

4. Fife, K.H. et al. Vaccine 22, 2943-2952 (2002).

5. Koutsky, L.A. et al. N. Engl. J. Med. 347, 1645-1651 (2002).

6. Clark, H.F. et al. Pediatr. Infect. Dis. J. 22, 14-20 (2003).

7. Clark, H.F. et al. J. Pediatr. 144, 184-190 (2004).

8. Pisano, G.P. in The Development Factory (Harvard Business School Press, Boston, 1997). 